\title{
Millimeter-wave Human Blockage Model Enhancements for Directional Antennas and Multiple Blockers
}

\author{
Danaisy Prado-Alvarez, Student Member, IEEE, Saúl Inca, \\ David Martín-Sacristán and Jose F. Monserrat, Senior, IEEE
}

\begin{abstract}
The Third Generation Partnership Project (3GPP) has defined a blockage model as an add-on feature to the channel model used in its evaluations of the physical layer in the 0.5-100 GHz frequency range. This paper focuses on the human body blockage losses in the millimeter-wave band proposing: (i) a new criterion to place the diffraction points over the edges of the blockers that considers the precise position of the blocker with regard to the line-of-sight between the transmitter and the receiver, (ii) a specific criterion to determine which potential blockers, from a set of human bodies present in a certain scenario, should be considered effective blockers given the positions of a transmitter, a receiver, and those bodies, and (iii) a modification of the model to provide more accurate estimations in the case in which several blockers are closely located. The validity of our criteria and the accuracy improvement are confirmed by a set of measurements performed at $30 \mathrm{GHz}$ with directional antennas and with multiple human blockers in different positions.
\end{abstract}

Index Terms-Body blockage model, multiple blockers, millimeter-wave, 3GPP

\section{INTRODUCTION}

$\mathbf{T}$ HE blockage effect at millimeter-wave (mmW) band has been a reason of concern due to the difficulty to transmit effectively in non line of sight (NLoS) conditions at these frequencies. One of the elements that blocks the $\mathrm{mmW}$ signals is the human body. In fact, in open-space indoor environments, typically with high human density, it can be the most important blocker. Indeed, it is important to model accurately each blocker but also to consider appropriately the presence of multiple blockers. For example, in [1], Jacob et al. presented an extension of the ray tracing (RT) based stochastic human blockage model for the IEEE 802.11ad standard at $60 \mathrm{GHz}$ which considers the presence of up to 10 users, while the original model only considered one user. The estimations of the model are more realistic in e.g., conference rooms.

Danaisy Prado-Alvarez, Saul Inca, and Jose F. Monserrat are with the iTEAM Research Institute, Universitat Politècnica de València, Camino de Vera s/n, 46022 Valencia, Spain e-mail: dapraal,sauin,jomondel@iteam.upv.es

David Martín-Sacristán is with Fivecomm, Camino de Vera s/n, 46022 Valencia, Spain e-mail: david.martin-sacristan@ fivecomm.eu

The work of Danaisy Prado was supported by the H2020 Marie Curie Program, with Project Grant No. 766231 WAVECOMBE - ITN - 2017. Part of this work has been performed in the framework of the $\mathrm{H} 2020$ project 5G-SMART co-funded by the EU. Saul Inca and Jose F. Monserrat would like to acknowledge the contributions of their colleagues from 5G-SMART although the views expressed are those of the authors and do not necessarily represent the views of the 5G-SMART project. This was also supported by the Spanish Ministry of Science, Innovation and University under the project RTI2018- 099880-B-C31.
Given this importance, a part of the research community has been recently focused on improving the human blockage models to get more realistic results. In [2], a human-body blockage model based on the doble knife-edge diffraction (DKED) is presented. This model offers a simple numerical representation for calculating the body blockage losses. Moreover, the 3GPP has incorporated the research conducted in this field to define a blockage modeling as an add-on feature to the channel model in [3]. This channel model is used in the $3 \mathrm{GPP}$ evaluations of the physical layer in the $0.5-100 \mathrm{GHz}$ frequency range. The body blockage model considers two variants: one based on a stochastic method and the other based on a geometric method. The first model (Model A) can be used in cases where a generic modeling is enough to capture human and vehicular blocking. Due to its simplicity, this model does not demand a high computational cost. The second model (Model B), inspired in the model presented in [2], was designed for those cases where a more realistic modeling of the scenario and the blockers is needed. Nevertheless, the computational complexity of this model is still much lower than in the case of a RT approach [4], where several rays need to be launched to mimic the signal propagation in real life. In Model B, the human bodies in a scenario are considered blockers of a link between a transmitter (TX) and a receiver (RX) based on a proximity criterion. Specifically, only those close to the RX are considered blockers. Then, the blockers are modeled as rectangular screens with one diffraction point per edge, positioned in the middle of each side, and their contributions are calculated in an isolated manner. Finally, the contributions from each blocker are summed.

The Mobile and wireless communications Enablers for Twenty-twenty Information Society (METIS)/3GPP model has been studied, e.g., in [5]. This work proposed an adaptation of the model that takes into account the use of directional antennas.

This letter presents a series of modifications and criteria to improve the accuracy of the 3GPP model without significantly increasing its complexity. Firstly, it proposes to relocate the diffraction points in such a way that their position depends on the heights and positions of the antennas and the screen. Secondly, it suggests to determine the blockage area to effectively define the users (human bodies) that should be considered as blockers. Finally, in case of multiple blockers, when they are in close proximity, this letter proposes to model them as a single body with a width equal to the product of the 
number of close blockers and the width of a single blocker. To validate these proposals, a measurement campaign is carried out. The results of the original $3 \mathrm{GPP}$ model are compared to these modifications, which show an increased accuracy.

The remainder of the paper is as follows. Section II exposes the proposals of this letter for the 3GPP body blockage model improvement. Section III describes the designed setup used in an indoor measurement campaign at $30 \mathrm{GHz}$. Section IV shows and analyzes the results of the measurement campaign and the predictions of the 3GPP model with and without our modifications. Finally, the conclusions are drawn in Section V.

\section{Proposals For 3GPP Body BlockAGE Model IMPROVEMENT}

\section{A. Re-position of the Diffraction Points}

In the 3GPP blockage model, the human blockers are modeled as rectangular screens with its normal vector parallel to the floor and oriented towards the TX. The infinite set of real diffraction points around the object are then discretized, for the sake of simplicity, leaving a central one at each edge of the screen (see in Fig. 1, point D).

This model is totally valid when omnidirectional antennas are considered or, in general, when a signal beam coming from a TX antenna hits the blocker in its full amplitude. However, cases where this beam does not cover the entire screen may occur, especially when working with directional antennas at a relatively short distance. In such cases, calculating the diffraction points based on the location of the antennas and the beam pointing would be more accurate. In fact, we propose to take as diffraction points those lateral points that are at the height where the central components of the beam emitted from the TX antenna are impacting (see in Fig. 1, point D').



Fig. 1. Re-position of diffraction points (side view).

\section{B. Blockage Area Determination}

In order to apply the 3GPP body blockage model in a scenario for a specific TX - RX link, the first step is to determine which elements of the scenario are considered as blockers. The criterion from the 3GPP is to consider as blockers those elements which are "close" or at a "certain distance" from the RX. The drawback of this criterion is obvious, as it does not provide an objective criterion to be followed in every situation. In addition, this criterion may be valid in scenarios with high-height TX antennas, but if the TX antenna height is low, a human close to the TX may also block the signal directed towards a distant RX. The latter may be the case for scenarios where device-device communications are considered. To provide a more clear determination, valid also in scenarios with low-height antennas, we propose to use the concept and calculation procedure of blockage zone presented in [6]. In that paper, the blockage zone concept is used as a criterion to determine if a moving object is blocking the radio signal to be able to model the system capacity in a random field of moving potential blockers. The contribution of this paper is to use this concept integrated with the 3GPP channel model.

For the sake of completeness, we provide an explanation of the blockage area concept in the following lines. Given a specific location and height of a TX and a RX, and a specific combination of width and height for potential blockers, in [6] the blockage area is defined as a region around the RX where any potential blocker would be effectively blocking the line of sight (LoS) from the TX. The concept is illustrated in Fig. 2. A blockage area is shown as a shaded area between the TX and the RX. Two potential blockers are shown as cylinders. The cylinder whose axis falls within the blockage area is considered as blocker, while the other whose axis is out of the blockage area is not considered as blocker. We propose to use a simplified rectangular shape for the blockage area as shown in Fig. 2, following the approach of [6]. Specifically, given a body width, the blockage area can be modeled as a rectangular region of body width $\mathrm{x} R_{\text {blockage }}$ squared meters, where $R_{\text {blockage }}$ can be calculated with the following equation:

$$
R_{\text {blockage }}= \begin{cases}d_{T X^{\prime}-R X^{\prime}}\left(\frac{h_{\text {body }}-h_{R X}}{h_{T X}-h_{R X}}\right) & \text { if } h_{T X}>h_{R X} \\ d_{T X^{\prime}-R X^{\prime}} & \text { if } h_{T X}=h_{R X}\end{cases}
$$

where $T X^{\prime}$ and $R X^{\prime}$ are the projections of $T X$ and $R X$ onto the floor plane, $d_{T X^{\prime}-R X^{\prime}}$ is the distance between $T X^{\prime}$ and $R X^{\prime}$, and $h_{T X}, h_{R X}$ and $h_{b o d y}$ are the heights of the TX, RX and blocker, respectively (see Fig. 2). Cases where the TX height is less than the RX height are not considered.

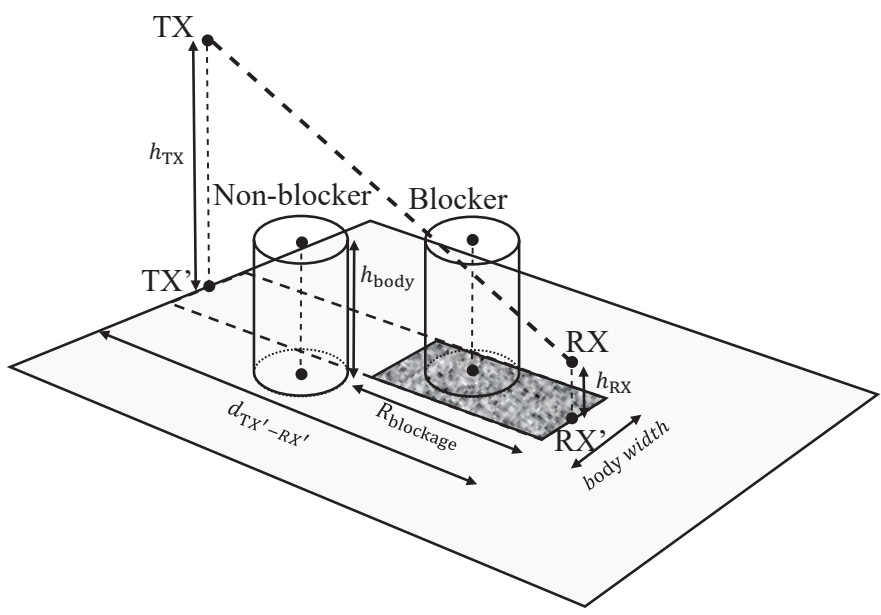

Fig. 2. Blockage area determination

\section{Close Blockers Grouping}

When multiple blockers are considered, it may be the case where the link from the diffraction points of some of them to the RX are also blocked by other blockers. In that case, the 
blocking losses may be higher than the values provided by the 3 GPP because in its model the contributions are calculated separately for each blocker and summed.

This letter focuses on a specific case in which various blockers are so close that they are perceived as a single body of greater width. What it is proposed in that case is to include them in the 3GPP model as a body $K$ times wider, being $K$ the number of blockers close to each other. Two bodies can be modeled as a single body if the cylinders modeling them overlap or are, at least, side by side (see Fig. 3).

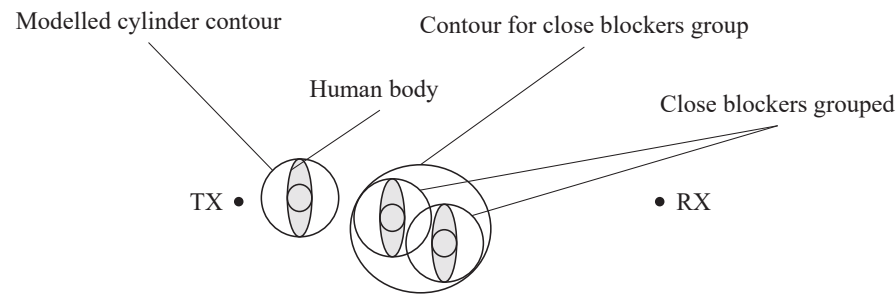

Fig. 3. Grouping of close blockers

\section{Scenario And SETUP FOR MeAsurements}

\section{A. Scenario}

The measurement scenario is an indoor open space. The distance between the TX and the RX is $4 \mathrm{~m}$ and their height is $1.2 \mathrm{~m}$, similarly to previous works [5], [7]. Fig. 4 illustrates this scenario. The measurements were carried out for one, two and three human blockers. For the case of one blocker only (Case 1), this one was positioned facing the RX antenna, over the TX'-RX' line, at 7-different locations going from $0.5 \mathrm{~m}$ to $3.5 \mathrm{~m}$ from the TX with steps of $0.5 \mathrm{~m}$. For Case 2 and Case 3, two and three humans respectively were positioned side by side with the same orientation and locations considered in Case 1. In Case 2, the two humans are positioned as close as possible and the edge between the bodies is over the TX'-RX' line. In Case 3, the middle blocker is over that TX'-RX' line. Cases 2.1 and 3.1 are modifications of Case 2 and Case 3. In both cases, a blocker is in the same locations of the single blocker of Case 1. The second blocker is positioned just after the first blocker with a half body shift from the TX'-RX' line. Whereas, in Case 3.1, the third blocker is also positioned after the first blocker but presents half body shift from the TX'-RX' line in the opposite direction of the second blocker shift. The measurements are repeated for all cases, alternating the participants, with the objective of obtaining consistent results. Table. III-A presents the physical characteristics of the three volunteers and their participation in each measurement case. For each specific measurement, the measurement duration is 4 seconds, generating 100,000 samples from which we obtain the median value.

TABLE I

VOLUNTEER INFORMATION

\begin{tabular}{|c|c|c|c|c|}
\hline Id & Breadth [m] & Depth [m] & Height [m] & Case involvement \\
\hline 1 & 0.39 & 0.21 & 1.81 & Case 1,3,3.1 \\
2 & 0.43 & 0.24 & 1.70 & Case 2, 2.1,3,3.1 \\
3 & 0.42 & 0.25 & 1.73 & Case 2, 2.1, 3, 3.1 \\
\hline
\end{tabular}

\section{B. Setup}

Fig. 4 illustrates the measurement setup used in this assessment to obtain body blockage losses measurements at $30 \mathrm{GHz}$. A vector network analyzer (VNA) is used to measure the losses between the transmitted and the received signals. The TX antenna is connected to the port 1 of the VNA and an amplifier is connected between the port 2 and the $\mathrm{RX}$ antenna. The VNA is the ZNB40 of the manufacturer Rhode\&Schwarz, with a working frequency range between $100 \mathrm{MHz}$ and $40 \mathrm{GHz}$. The output power is fixed to $0 \mathrm{dBm}$ in our measurements. The amplifier is a SHF 810 with a gain of $29 \mathrm{~dB}$. The antennas are identical WRD180 wideband horn antennas, with $14.1 \mathrm{dBi}$ of maximum gain and a $3-\mathrm{dB}$ beamwidth of 30 degrees at $30 \mathrm{GHz}$ [8]. According to the antenna characteristics and the work frequency, the far field distance is approximately $0.25 \mathrm{~m}$, so it can be assured that the signal is received in the far field for all cases considered in this work.

\section{RESUlTS AND ANALYSIS}

This section compares the measurement results, obtained in the scenario presented in the previous section, with the predictions of the 3GPP human body blockage model with and without our proposed modifications related to the positioning of the diffraction points and the modeling of close blockers. In all the cases the 3GPP model was extended following the approach in [5] to consider directional antennas. Therefore, the technical specifications of the antennas used in our measurements were considered in the computation of losses. Specifically, the half power beamwidth of the antennas involved has been used in the equation 5 from [5] to obtain the parameters $a$ and $b$, then the gain of antennas has been computed and finally used in the equation 6 from [5] to determine the body blockage losses.

Concerning the criterion to determine which bodies are signal blockers, following the blockage area determination method explained in Section II-B, all the human bodies involved in the measurements should be considered blockers except the two side bodies in Case 3. The reason is that given that the heights of the TX and RX are equal, the blockage area extends from the TX to the RX as shown in Fig. 5. Therefore, all the potential blockers can be effectively blocking the signal irrespective of their distance to the TX or RX, contrarily to what is stated by the 3GPP model. On the other hand, the width of the blockage area is equal to the width of a potential blocker. As a result, the side bodies in Case 3 are out of this region and should not be considered effective blockers.

In the 3GPP blockage model, the human blockers are modeled as rectangular screens with a height of $1.7 \mathrm{~m}$ with its normal vector parallel to the floor and oriented towards the TX. The diffraction points in the left and right sides of the screen are located in the middle of the side edge, i.e. at a height of $0.85 \mathrm{~m}$. In our setup, the TX and RX antennas are positioned at a height of $1.2 \mathrm{~m}$, therefore, the TX-RX line-of-sight cuts the screen at a height of $1.2 \mathrm{~m}$. In order to analyze the impact of the precise position of the diffraction points, the loss predicted by the $3 \mathrm{GPP}$ model is compared with side diffraction points at 


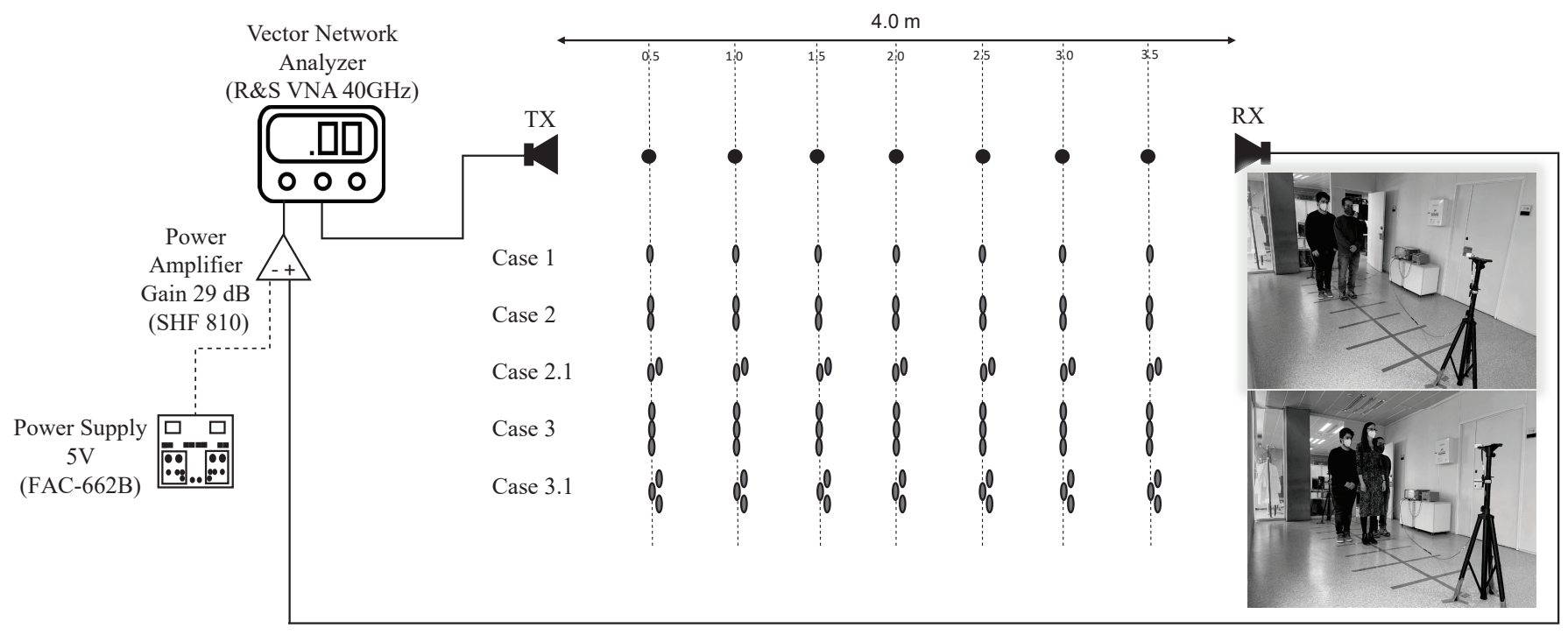

Fig. 4. Setup and measurement cases. Case 2.1 (upper picture), Case 3.1 (lower picture)



Fig. 5. Zenithal view of the blockage area in our measurement setup

$0.85 \mathrm{~m}$, as in the original model, and at $1.2 \mathrm{~m}$, as proposed in Section II, with the results of real measurements considering a single blocker, i.e. focusing on the Case 1 explained in the previous section.

The results are shown in Fig. 6, where the body blockage losses for Case 1 and Case 3 are represented in function of the distance from the blockers to the RX. The real measurements for one blocker are denoted as "Measurement (one blocker)", the 3GPP model without modifications as "3GPP (one blocker)", and the 3GPP model with the re-positioning of diffraction points (RDP) as "3GPP+RDP (one blocker)". The mean absolute error for the $3 \mathrm{GPP}$ model is $8.21 \mathrm{~dB}$, while the error is $2.05 \mathrm{~dB}$ with the proposed modification. This result reinforces this approach. In Case 3, the application of the method proposed in this letter to determine which human bodies should be considered as blockers leads to the consideration of only one blocker, concretely, the central human body. For the sake of comparison, measurements with the Case 3 setup are included ("Measurement (three blockers)") as well as the results when the three bodies are considered blockers as "3GPP (three blockers)" for the original model (with the original diffraction points) and "3GPP + RDP (three blockers)" for the 3GPP model with the re-position of diffraction points. Results demonstrate the validity of the proposed approach to determine the bodies that should be considered blockers and corroborates the increased accuracy provided by the discussed positioning of the diffraction points.

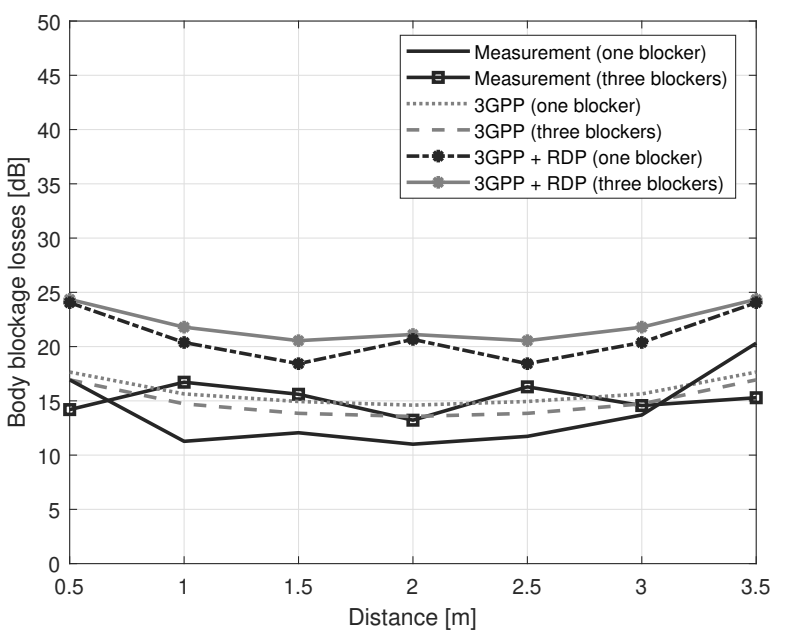

Fig. 6. Body blockage losses: Case 1 (one blocker) and Case 3 (three blockers in parallel)

In Case 2, Case 2.1 and Case 3.1, the application of the criterion proposed in this letter to determine the blockers leads to more than one blocker being considered. Therefore, in these cases the validity of the proposal for close blockers grouping (CBG) presented in Section II-C was analyzed. The results of the measurements and the model calculations are shown in Fig. 7, Fig. 8 and Fig. 9, respectively. As in the previous cases, it is depicted the result of the measurements ("Measurement"); the original 3GPP model ("3GPP") with an absolute error with respect to measurements of $27.77 \mathrm{~dB}$ for Case 2, $20.16 \mathrm{~dB}$ for Case 2.1 and $4.03 \mathrm{~dB}$ for Case 3.1; the result of modifying the diffraction points location ("3GPP + RDP") with an absolute error of $4.17 \mathrm{~dB}$ for Case 2, $3.53 \mathrm{~dB}$ for Case 2.1 and $7.85 \mathrm{~dB}$ for Case 3.1; and the result of also grouping closely spaced bodies ("3GPP + RDP + CBG") with an absolute error of $6.34 \mathrm{~dB}$ for Case 2, $4.03 \mathrm{~dB}$ for Case 2.1 and $2.98 \mathrm{~dB}$ for Case 3.1. Considering that in general the results of " $3 \mathrm{GPP}$ 
+ RDP" are optimistic and the results of "3GPP + RDP + CBG" are pessimistic, an additional graph ("avg(3GPP + RDP, $3 \mathrm{GPP}+\mathrm{RDP}+\mathrm{CBG}$ )") has been also included presenting the average result of the two last approaches, i.e., the average losses of the cases with grouping and without grouping of blockers. This is precisely the approach that provides the most accurate results in all the cases (absolute error equal to $1.28 \mathrm{~dB}, 3.62 \mathrm{~dB}$ and $2.84 \mathrm{~dB}$ for Case 2, Case 2.1 and Case 3.1 respectively) and the one we propose to be followed in case of having close blockers in a scenario.

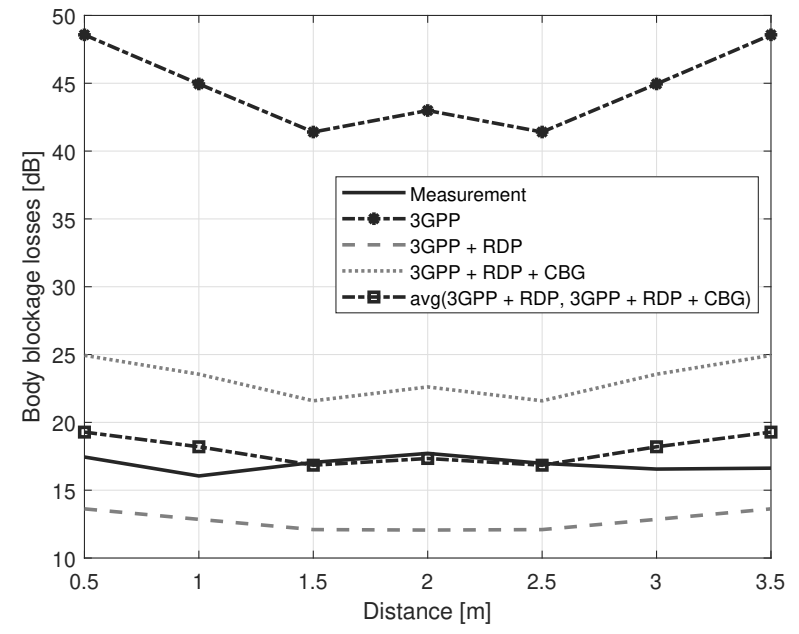

Fig. 7. Body blockage losses: Case 2

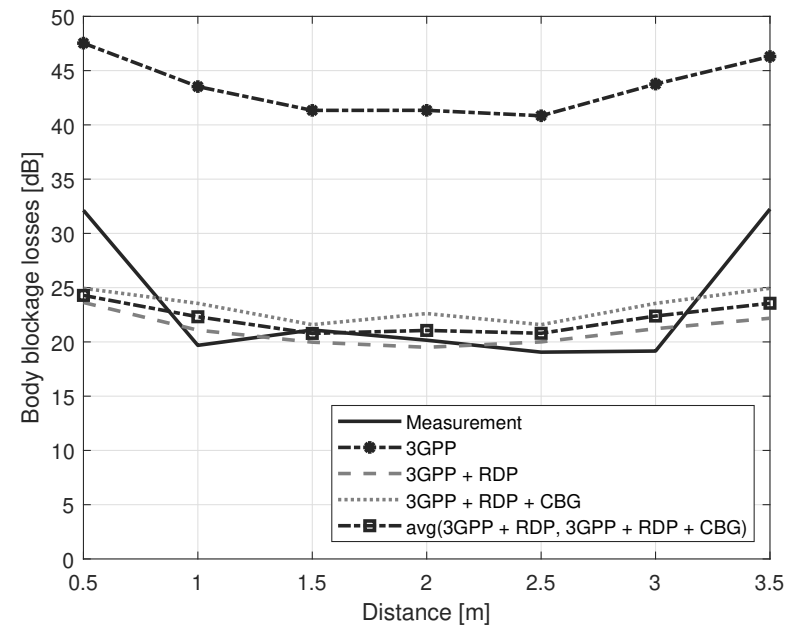

Fig. 8. Body blockage losses: Case 2.1

\section{CONCLUSIONS}

This letter has presented a measurement campaign in order to compare the results with those from the 3GPP body blockage model. Five specific cases of body blockage were described considering one, two and three human blockers obstructing the LoS between the TX and the RX. As a modification for the human body modeling of the 3GPP, the re-positioning of the side diffraction points according to the TX and RX height is proposed, demonstrating that more

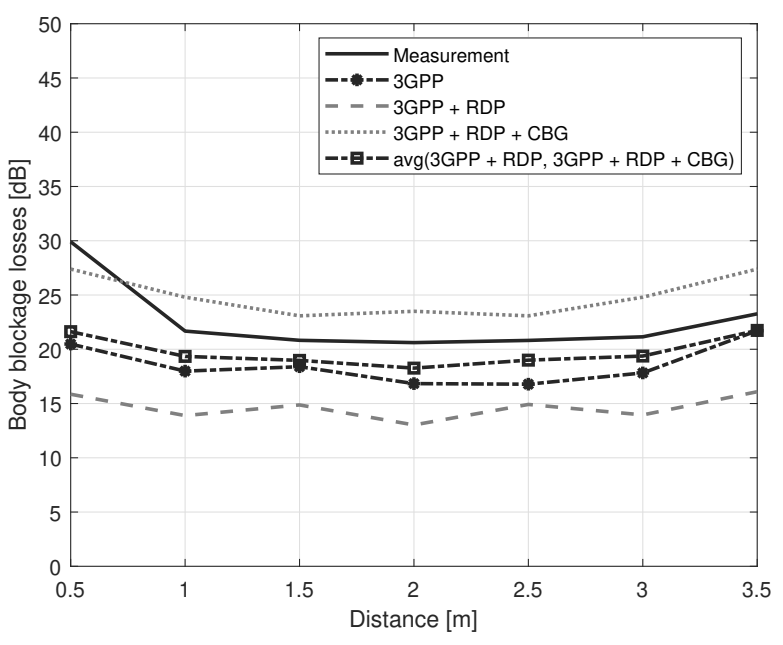

Fig. 9. Body blockage losses: Case 3.1

accurate results can be achieved with this modification while keeping simplicity. For the determination of which potential blockers should be considered effective blockers, it has been provided a criterion based on the definition of a blockage area calculated for a RX, proving that following this criterion it is possible to get accurate results. In addition, this criterion is more clear than the one provided by the 3GPP. Although, the scenario was set up for equal heights for the TX and RX, the blockage area determination method is applicable also for different heights of the TX and RX. These conclusions can be useful for the research community since they take the burden off of having to decide which are the closest users of RX in order to take into account the attenuation caused by these. Finally, in case of having closely spaced blockers, the loss prediction provided by the 3GPP model can be improved by averaging them with a modification of that model in which the closely spaced blockers are modeled as a single body with increased size. This work will be extended to larger scenarios with more blockers.

\section{REFERENCES}

[1] M. Jacob, S. Priebe, T. Kürner, M. Peter, M. Wisotzki, R. Felbecker, and W. Keusgen, "Extension and Validation of the IEEE 802.11ad $60 \mathrm{GHz}$ Human Blockage Model," in 2013 7th European Conference on Antennas and Propagation (EuCAP), 2013, pp. 2806-2810.

[2] METIS2020, "METIS Channel Model," METIS2020, Tech. Rep., Deliverable D1.4 v3, July 2015.

[3] ETSI, "5G; Study on channel model for frequencies from 0.5 to $100 \mathrm{GHz}$ (3GPP TR 38.901 version 14.0.0 Release 14)," 3GPP, Tech. Rep., 2017.

[4] M. Nakamura, M. Sasaki, N. Kita, and Y. Takatori, "Path Loss Model in Crowded Areas considering Multiple Human Blockage at 4.7 and $26.4 \mathrm{GHz}$," in 2017 IEEE Conference on Antenna Measurements \& Applications (CAMA). IEEE, Dec. 2017.

[5] G. R. MacCartney, S. Deng, S. Sun, and T. S. Rappaport, "Millimeter-Wave Human Blockage at $73 \mathrm{GHz}$ with a Simple Double Knife-Edge Diffraction Model and Extension for Directional Antennas," in 2016 IEEE 84th Vehicular Technology Conference (VTC-Fall). IEEE, Sept. 2016.

[6] D. Moltchanov, A. Ometov, S. Andreev, and Y. Koucheryavy, "Upper Bound on Capacity of $5 \mathrm{G} \mathrm{mmWave} \mathrm{Cellular} \mathrm{with} \mathrm{Multi-Connectivity}$ Capabilities," Electronics Letters, vol. 54, no. 11, pp. 724-726, May 2018.

[7] U. T. Virk and K. Haneda, "Modeling Human Blockage at 5G Millimeter-Wave Frequencies," IEEE Transactions on Antennas and Propagation, vol. 68, no. 3, pp. 2256-2266, Mar. 2020.

[8] Steatite, "Wideband Horn Antenna 18 to $40 \mathrm{GHz}$ WRD180," Datasheet. 